\title{
Genes and Environment in BRCA-Positive Breast Cancer
}

\author{
Colin A. Ross, M.D. \\ The Colin A. Ross Institute for Psychological Trauma \\ 1701 Gateway, \#349 Richardson, TX 75080, USA \\ rossinst@rossinst.com
}

\begin{abstract}
The risk of developing breast cancer by age 50 in BRCA-positive women has increased from $24 \%$ in women born before 1940 to $67 \%$ in women born after 1940. This observation points to an increase in one or more environmental promoters through the course of the twentieth century. Extrapolating backwards, it is possible that, in the nineteenth century, a BRCA mutation conferred minimal increased risk for breast cancer. A testable model for gene-environment interactions in BRCA-positive women is proposed that may be generalizable to other cancers. A shift in funding emphasis from studying genes in isolation to studying them in conjunction with environmental promoters is proposed.
\end{abstract}

Keywords: breast cancer, genetic causation, environmental causation, BRCA gen

\section{INTRODUCTION}

A great deal of research is currently devoted to studying the genetics of cancer, including risk genes for breast cancer, of which the most prominent are the BRCA1 and BRCA2 mutations. In this regard, the National Cancer Institute states on its web site that, "About 12 percent of women in the general population will develop breast cancer sometime during their lives. By contrast, according to the most recent estimates, 55 to 65 percent of women who inherit a harmful BRCA1 mutation and around 45 percent of women who inherit a harmful BRCA2 mutation will develop breast cancer by age 70 years" (http://www.cancer.gov/about-cancer/causes-prevention/genetics/brca-fact-sheet).

It might appear from these statistics that the BRCA gene causes breast cancer, however, this is not actually the case. The BRCA gene is a tumor suppressor gene. Its function is to repair damage to DNA [1-3]. When a mutation renders the gene partially or completely non-functional, damage to DNA is not repaired and cancer is much more likely to develop. Why the BRCA mutations do not increase the risk for cancer throughout the body is unknown, but there is no question that they increase the risk for breast and ovarian cancer.

What model best fits what we know about the role of genes and environment in women with a BRCA mutation? The BRCA gene does not directly cause cancer, so a simple unidirectional model cannot apply.

\section{The Marie-Claire King BrCa Study}

King, Mark and Mandell [2] studied 1008 Ashkenazi Jewish women with a BRCA mutation. The authors divided their sample into women born before or after 1940, and a most intriguing observation was made. Among BRCA-positive women born after 1940, the risk of developing breast cancer by age 50 was $67 \%$. However, among those women born before 1940, it was only $24 \%$.

This observation should be replicated in multiple different samples. However, assuming that it is valid and generalizable, there are only three possible explanations for it: 1) something in the environment has been driving an increase in breast cancer over the twentieth century, 2) there has been a pronounced shift in the predominant type of BRCA gene mutation, resulting in greater gene penetrance, or 3) a combination of (1) and (2) is operating.

It seems unlikely that such a pronounced shift in the genome has occurred by random mutation in one or two generations, therefore it seems likely that one or more promoters has become more prevalent in the environment. Whatever these promoters are, it is possible that if we could extrapolate backwards into the nineteenth century (which we can't), the rate of breast cancer in BRCA-positive women might be the same as the base rate in the general population, or only a few percentage points higher. 
Additionally, the base rate in the general population might be lower than the $12 \%$ rate cited by the National Cancer Institute.

\section{A Model of Genes and Environment in BrCa-Positive Breast Cancer}

I propose a scientifically testable model of gene-environment interaction resulting in breast cancer in BRCA-positive women. In this model, in the majority of cases, one or more environmental promoters are required to cause DNA damage. Once the damage has occurred, in the majority of women a functional BRCA gene repairs the DNA and no cancer develops. In women with a BRCA mutation however, DNA repair is markedly less successful, and breast cancer is much more likely to develop, as is ovarian cancer. The results in the following risk groups for breast cancer:

BRCA mutation - high environmental promoter level:

high risk

BRCA mutation - intermediate environmental promoter level: intermediate risk

BRCA mutation - low environmental promoter level:

low risk

No BRCA mutation - high environmental promoter level:

intermediate risk

No BRCA mutation - intermediate environmental promoter level: low risk

No BRCA mutation - low environmental promoter level:

low risk

Two questions follow from this model: 1) what are the known and suspected environmental promoters? and 2) what percentage of funding should be allocated to genetic studies, and what percentage to environmental studies?

I propose that the allocation of breast cancer research funding is markedly out of balance. The King, Mark and Mandell [2] findings suggest that the risk for breast cancer in BRCA-positive women could be reduced by $(67-24 / 67 \times 100 \%=64 \%)$ if the environmental promoter levels could be reduced to those in the first half of the twentieth century. If the promoter levels were even lower in the nineteenth century, the potential reduction in breast cancer rates through environmental modifications could exceed $80 \%$ or $90 \%$. I propose that the allocation of breast cancer research funding should therefore be shifted towards identification of environmental promoters and their mechanisms of action.

\section{Known and Suspected Environmental Promoters of Breast Cancer}

King, Mark and Mandell [2] noted that physical exercise in adolescence and lack of obesity delayed the onset of breast cancer in their sample. Besides BRCA and other less important gene mutations [3], the identified risk factors for breast cancer include: family history, obesity, exposure to exogenous estrogens after menopause, lack of exercise, obstetrical and breast-feeding history [1-4], and a combination of obesity and low vitamin D [5]. Some sort of industrial pollution might play a role as well, through chemicals in our food, air or water.

The effect of any environmental promoter could be studied in animal models. Animals with a known DNA-repair mutation and an increased risk for cancer could be exposed to varying doses and combinations of promoters with their rate of developing cancer as the outcome variable. Several of these promoters could exist on a continuum and act as promoters at one end (obesity) and protectors (low body weight) at the other end. The main point is to study the genes and the environment in concert, not as isolated variables. Within this model, which may apply to a range of different cancers, there are no genes for breast cancer as such, rather there are genes that confer a vulnerability to the carcinogenic effect of environmental promoters. This model, I propose, applies to a large percentage of cancers. However, in a certain number of cases, gene penetrance might be so high in the absence of environmental promoters, that the risk of cancer is high without a requirement for a carcinogen. From a public health perspective, I am proposing, this latter pathway to cancer is smaller in size than the gene-environment interaction model I have described.

In humans, studies could attempt to find populations with and without known risk genes, and with low and high exposure to environmental promoters, and could then track the cancer rates in the different subgroups. Finally, interventions such as weight reduction, increased vitamin D intake, and a comparison of synthetic to bio-identical post-menopausal hormone replacement could be conducted.

Campbell [6] has provided a two-strike model of environmental promoters that illustrates how more than one promoter might act synergistically. In his mouse model, animals exposed to a known 
carcinogen via its application to their skin had a reduced rate of developing tumors if they were fed a diet low in animal protein and high in plant protein. Campbell [6] studied the biochemistry of these effects over a period of decades and also conducted large-scale epidemiological studies in humans [7].

\section{Conclusions}

If the observation of King, Mark, and Mandell [1] is valid and generalizable, then the risk for breast cancer in BRCA-positive women may be driven predominantly by the environment, not by the genome. It follows that, if this is true, there should be a shift in cancer research funding away from studying genes in isolation, and towards studying their effects in both the presence and the absence of carcinogenic environmental promoters.

\section{REFERENCES}

[1]. Antoniou A., Pharaoah P.D., Narod S., et al. Average risks of breast and ovarian cancer associated with BRCA1 or BRCA2 mutations detected in case series unselected for family history: a combined analysis of 22 studies. Am. J. Hum. Genet. 72, 1117 (2003).

[2]. King M.-C., Mark J.H., Mandell J.B. Breast and ovarian cancer risks due to inherited mutations in BRCA1 and BRCA2. Science. 302, 643 (2003).

[3]. Cornejo-Moreni B.A., Uribe-Escamilla D., Salamanca-Gomez F. Breast cancer genes: looking for BRACA's lost brother. Isr. Med. Assoc. J. 16, 787 (2014).

[4]. Donepudi M.S., Kondapalli K., Amos S.J., et al. Breast cancer statistics and markers.

[5]. Duggan C., Tapsoba J. de D., Mason C., et al. Effect of vitamin D3 supplementation in combination with weight loss on inflammatory biomarkers in postmenopausal women: A randomized controlled trial. Cancer Prev. Res. 8, 28 (2015). J. Cancer Res. Ther. 10, 506 (2014).

[6]. Campbell T.C. Dietary protein, growth factors, and cancer. Lancet. 85, 1667 (2007).

[7]. Campbel T.M., Campbell T.C. The China Study: Startling Implications for Diet, Weight Loss, and Long-Term Health. Dallas, TX: BenBella Books, 2005.

\section{AUTHOR's BIOGRAPHY}



Dr. Ross, is an internationally renowned clinician, researcher, author and lecturer in the field of traumatic stress and trauma related disorders. He is the founder and President of the Colin A. Ross Institute for Psychological Trauma. Dr. Ross is also the Executive Medical Consultant of three trauma programs located in Dallas, Texas, Grand Rapids, Michigan and Torrance, California. In addition to his treatment programs Dr. Ross has authored over 180 professional papers and 28 books. He is a Past President of the International Society for the Study of Trauma and Dissociation. Dr. Ross has written three papers about cancer and is planning a fourth. 